\title{
Massive Hemorrhage into the Iliopsoas Muscle
}

Key words: Hemophilia B, acute abdomen, bleeding into iliopsoas muscle

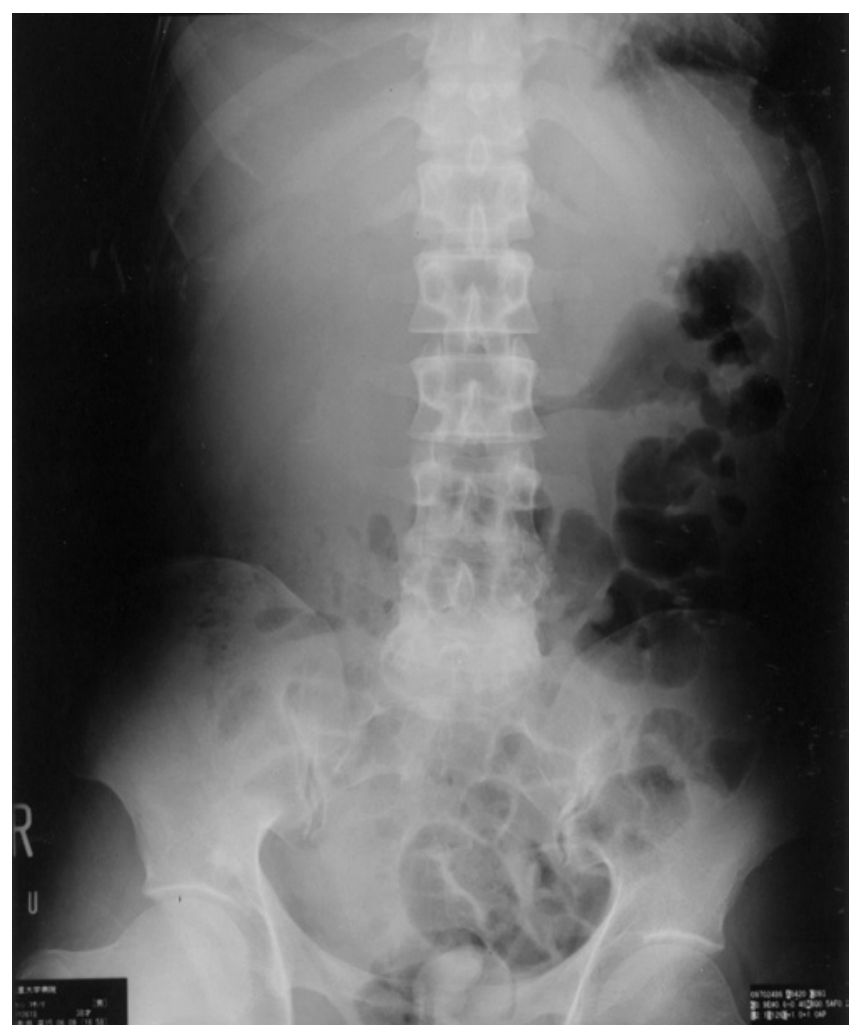

Figure 1.

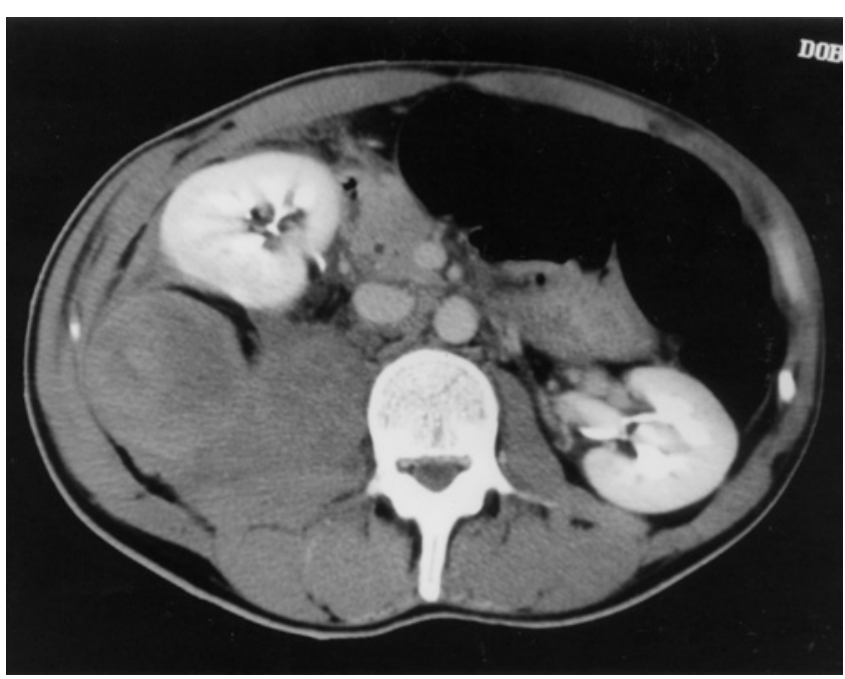

Figure 2.

A 38-year-old man with severe deficiency of factor IX (Hemophilia B) was admitted for right lower abdominal pain of progressively increasing severity and tenderness with muscular defense. He had suffered from a common cold with severe cough and loss of appetite for about a week. Abdominal radiograph showed the positive psoas sign on the right side and the left-shifted colon gas (Fig. 1). Computed tomography (Fig. 2) revealed massive hematoma in the right iliopsoas muscle, resulting in anteriorward translocation of the right kidney. The reconstructed 3-dimensional image (Fig. 3) demonstrated more clearly the kidney translocation and the extended but intact large vessels. These are useful findings for the diagnostic procedures, because progressive right lower abdominal pain may closely simulate acute appendicitis. The hemorrhage was successfully managed by replacing factor IX for a week without any recurrence. He did not have any inhibitors to factor IX. 


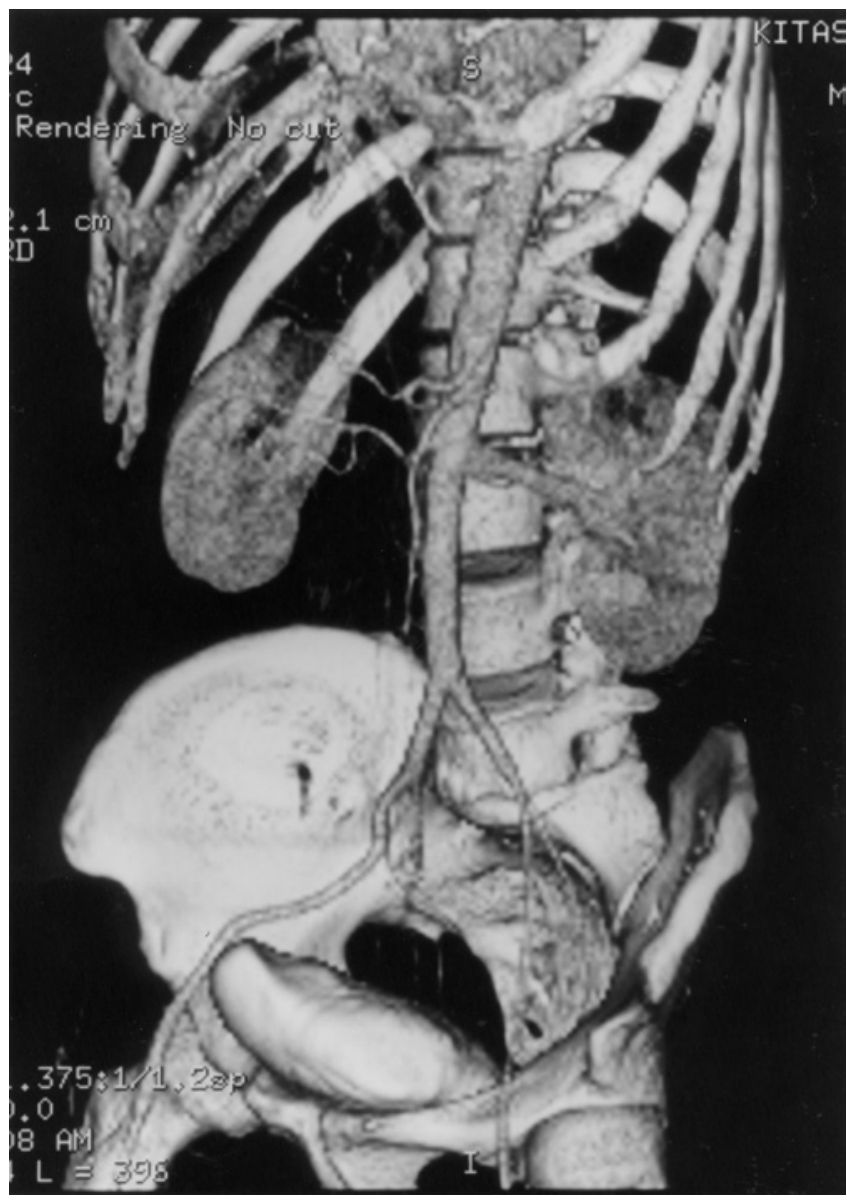

Figure 3.

Koji MiYAZAKI and Masaaki HigashiHARA

From the Fourth Department of Internal Medicine, Kitasato University School of Medicine, Sagamihara

Received for publication September 7, 2004; Accepted for publication October 1, 2004

Reprint requests should be addressed to Dr. Koji Miyazaki, the Fourth Department of Internal Medicine, Kitasato University School of Medicine, 1-15-1 Kitasato, Sagamihara, Kanagawa 228-8555 